\title{
Biokatalyse
}

\section{Pilzliche Peroxygenasen: der Schlüssel zu C-H-Hydroxylierungen und mehr?}

PASCAL PÜLLMANN, MARTIN J. WEISSENBORN

LEIBNIZ-INSTITUT FÜR PFLANZENBIOCHEMIE (IPB), HALLE (SAALE), INSTITUT FÜR CHEMIE, UNIVERSITÄT HALLE-WITTENBERG

Fungal peroxygenases represent an exciting new enzyme class for stereoselective hydroxylation reactions. They are capable of the oxyfunctionalisation of a large, diverse scope of substrates including alkanes and steroids as well as the heteroatoms sulfur and nitrogen. The outstanding activities and stabilities as well as their reliance on hydrogen peroxide as co-substrate renders it a highly interesting biocatalyst.

DOI: $10.1007 / \mathrm{s} 12268-019-1090-2$

(C) Die Autoren 2019
Die spezifische Aktivierung von C-H-Bindungen in einem Reaktionsschritt stellt eine der großen Herausforderungen der organischen Chemie dar. Eine effektive, direkte sowie stereoselektive Hydroxylierung von C-H-Bindungen könnte zahlreiche industriell relevante Synthesewege verkürzen und late stage functionalisations (LSF) ermöglichen. Die Enzymklasse der P450-Monooxygenasen
(Abb. 1A) ist in der Lage, eben diesen Reaktionstypus in wässriger Umgebung und bei Raumtemperatur chemo-, regio- und stereoselektiv zu katalysieren.

\section{Die neue Enzymklasse der} Peroxygenasen aus Basidiomyceten

Basidiomyceten (Ständerpilze) stellen eine interessante Quelle für biotechnologisch rele-

\section{A}

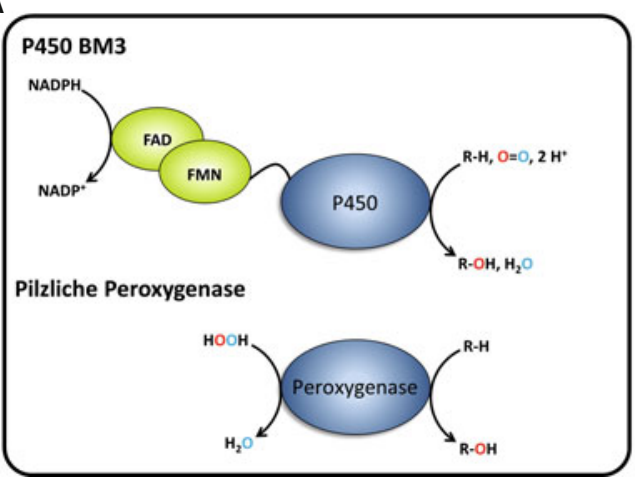

B

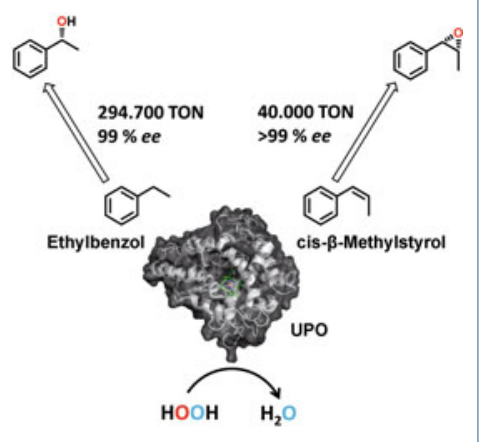

$\Delta$ Abb. 1: Vergleich von P450-Monooxygenasen und pilzlichen Peroxygenasen. A, P450-Monooxygenasen bestehen im Allgemeinen aus zwei bis drei assoziierten Proteinkomplexen, dem HämReaktionszentrum (P450) und einer NADPH-Cytochrom-P450-Oxidoreduktase (FAD). Bei den pilzlichen Peroxygenasen (long type-UPOs) liegt das Enzym als monomere Struktur vor. Der inkorporierte Sauerstoff entstammt dem Ko-Substrat Wasserstoffperoxid $\left(\mathrm{H}_{2} \mathrm{O}_{2}\right)$. FMN: Flavinmononukleotid. B, Beispielreaktionen von UPO aus Agrocybe aegerita (AaeUPO) mit einer hohen Aktivität sowie Enantioselektivität. Unter Verwendung von enzymatischen in situ-Wasserstoffperoxid-Generierungssystemen konnten für die Hydroxylierung von Ethylbenzol (links) sowie die Epoxidierung von cis- $\beta$-Methylstyrol (rechts) hohe turnover numbers (TON) erreicht werden (ee: enantiomere Überschüsse). vante Enzyme dar. Das Spektrum der prominenten, sekretierten Proteine umfasst unter anderem die Laccasen sowie diverse Peroxidasen und Oxidasen. Im Jahr 2004 haben Hofrichter und Mitarbeiter eine neue Enzymklasse aus dem Überstand des Pilzes Agrocybe aegerita charakterisiert [1]. Diese als unspezifische Peroxygenasen (UPO, E.C. 1.11.2) bezeichneten Enzyme sind in der Lage, ein enorm breites Substratspektrum effizient zu hydroxylieren. Dazu zählen unter anderem aromatische, benzylische und aliphatische Kohlenwasserstoffe (Abb. 2, [2, 3]). UPOs konnten benzylische Hydroxylierungen mit bis zu 300.000 TON (turnover number) katalysieren, ein beeindruckender Wert für eine enzymatische Hydroxylierung von $\mathrm{sp}^{3}$-Kohlenstoffatomen, und weisen dabei enantiomere Überschüsse (ee) von über 99 Prozent auf (Abb. 1B, [4]). Im Zuge der vermehrten Sequenzierung pilzlicher Genome konnten bereits über 4.000 putative PeroxygenaseGene annotiert werden, die sich breitflächig und funktional divers über die Reiche der Basidiomyceten sowie Ascomyceten verteilen [5].

\section{Heterologe Expression und Enzyme Engineering von Peroxygenasen}

Peroxygenasen sind stark glykosylierte sowie Disulfid-verbrückte Enzyme, sodass die funktionelle Expression dieser Enzymklasse nicht als trivial zu betrachten ist. Einen Meilenstein in der großflächigen Verbreitung der Peroxygenasen bilden die Arbeiten der Gruppe um Miquel Alcalde, CSIC Madrid [6], die im Rahmen eines gerichteten Evolutionsansatzes eine Peroxygenase-Variante entwickelt haben, die neun Mutationen im Vergleich zur Wildtyp-AaeUPO aufweist und als hochaktives Enzym in ausreichenden Mengen in dem eukaryotischen Modellorganismus Saccharomyces cerevisiae (Bäckerhefe) sekretiert wird.

\section{Gerichtete Evolution unabhängig von Farbassays}

Um eine gerichtete Evolution zu erlauben, arbeiteten wir zunächst an der Entwicklung eines effizienten Mutageneseverfahrens zur 
Erzeugung hoher genetischer Vielfalt. Die Golden Mutagenesis-Technik erlaubt die parallele Sättigungsmutagenese von bis zu fünf Positionen innerhalb des Zielgens unter Verwendung automatisch berechneter Primer (https://msbi.ipb-halle.de/GoldenMutagene sisWeb) [7].

Eine Limitation in den bisher durchgeführten gerichteten Evolutionskampagnen von UPOs stellt ein geeignetes Hochdurchsatzscreening dar. So basieren alle bisher durchgeführten Kampagnen auf der kolorimetrischen Detektion von Reaktionsprodukten im 96-Well-Format. Um das Spektrum der möglichen Substrate zu erweitern, arbeiten wir an einem Hochdurchsatz-GC-MS-Verfahren (MISER: multiple injection in a single experimental run) als Detektionsmethode. Die MISER-GC-MS-Analytik ermöglicht die Analyse eines breiten Spektrums an Reaktionsprodukten, basierend auf GC-Trennung und spezifischer Massenfragmentierung.

\section{Ein modulares Expressionssystem und neue Peroxygenasen}

Neben der Erschließung und Weiterentwicklung neuer Peroxygenase-Reaktivitäten ist außerdem der Zugang zu neuen Peroxygenase-Enzymen ein hochgradig relevanter Forschungsansatz. Wie bereits erwähnt sind putative Peroxygenase-Sequenzen, die eine hohe Sequenzhomologie zu dem Modellenzym AaeUPO aufweisen, über das Königreich der Pilze weitverbreitet. Vor dem Gesichtspunkt, dass eine effektive heterologe Herstellung der AaeUPO in S. cerevisiae erst nach intensiver Mutagenese gelingen konnte und vier der insgesamt neun eingebrachten Mutationen im für die Sekretion entscheidenden UPO-Signalpeptid lokalisiert sind, haben wir ein modulares Peroxygenase-Expressionssystem für die sekretierte UPO-Produktion in S. cerevisiae entwickelt [6]. Dieses System basiert auf der Golden Gate-Klonierungsmethode, die ein hocheffektives „one-pot onestep"-Klonieren von einzelnen Genabschnitten erlaubt [8]. Dazu wurde zunächst ein Golden Gate-kompatibles Hefe-Expressionsplasmid entwickelt, welches die PeroxygenaseExpression unter Kontrolle eines Galactoseinduzierbaren Promotors reguliert. Basierend auf dem augenscheinlich großen Einfluss des Signalpeptids auf eine erfolgreiche Peroxygenase-Produktion in Hefe haben wir innerhalb des modularen Systems ein Signalpeptidshuffling-System eingerichtet, das die zufallsverteilte Inkorporation verschiedener Signalpeptide ermöglicht, sodass geeignete Signal-

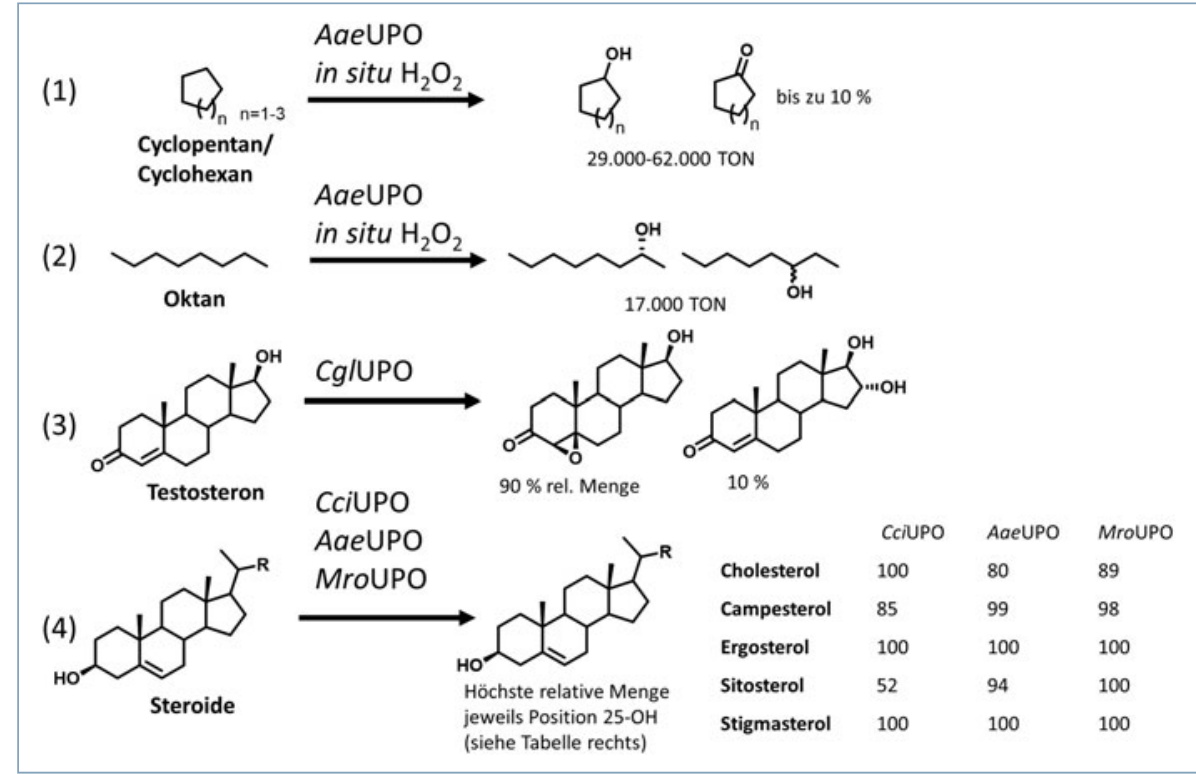

$\Delta$ Abb. 2: Aktivitäten und Spezifitäten unspezifischer Peroxygenasen (UPOs) mit besonderem Fokus auf selektiver Hydroxylierung nicht-aktivierter Kohlenstoffe. Die Reaktionen (1) und (2) wurden unter in situ-Wasserstoffperoxid-Generierung mit der Peroxygenase AaeUPO aus Agrocybe aegerita durchgeführt [4]. In Reaktion (3) konnte die Epoxidierung und respektive Hydroxylierung von Testosteron mit der Peroxygenase Cg/UPO aus Chaetomium globosum gezeigt werden [10]. Die Reaktion (4) zeigt ein Spektrum verschiedener Steroidderivate unter Testung der drei Peroxygenasen CciUPO, AaeUPO und MroUPO [11].

peptid-Peroxygenase-Kombinationen mit geringem Aufwand innerhalb einer einzelnen Screeningrunde identifiziert werden können.

Ein weiterer interessanter Faktor ist das Aktivitätsscreening, basierend auf klassischen Peroxidase- und Peroxygenase-Substraten. Möglicherweise weisen andere, putative Peroxygenasen keinerlei Aktivität gegenüber den Modellsubstraten oder unter den gewählten Konditionen auf, sodass sie als negatives Ergebnis innerhalb des Screenings auftreten würden. Um daher zwischen den Faktoren erfolgreiche Sekretion und enzymatische Aktivität unterscheiden zu können, beinhaltet das modulare Expressionssystem zudem einen C-terminalen Protein-Tag (27 Aminosäuren) zur split-GFP-basierten Proteindetektion. Dieser als GFP-11 bezeichnete Tag ist C-terminal mit der Peroxygenase fusioniert und bildet das elfte $\beta$-Faltblatt des grün fluoreszierenden Proteins (GFP) aus. In räumlicher Nähe zum komplementären restlichen GFP (GFP 1-10) wird zeitabhängig das Volllängen-GFP rekonstruiert und Fluoreszenz ausgebildet [9]. Die Zielproteinmenge ist dabei proportional zum sich ausbildenden Fluoreszenzsignal, das an einem Plattenlesegerät detektiert werden kann. Zudem kann die Peroxygenase direkt im Überstand detektiert werden und ein Lyse-Schritt, der häufig notwendig ist und einen zusätzlichen
Variationsparameter darstellt, entfällt. Außerdem bietet dieser als split-GFP bezeichneter Assay eine attraktive Möglichkeit der Normalisierung von der jeweiligen Enzymaktivität zur Proteinmenge im Falle von gerichteten Evolutionsansätzen, in welchen auch eine deutliche Variation der Produktionsmenge einzelner Varianten durch Mutagenese auftreten kann. Eine Übersicht des etablierten modularen Peroxygenase-Expressionssystems ist in Abbildung $\mathbf{3}$ dargestellt.

Wir sind zuversichtlich, mithilfe der entwickelten Methodenkombination aus modularem Expressionssystem sowie den Hochdurchsatzmethoden MISER-GC-MS und splitGFP das Portfolio an heterolog herstellbaren Peroxygenasen sowie das bisher bekannte Reaktionsspektrum durch Methoden der gerichteten Evolution zu erweitern.

\section{Danksagung}

Wir danken dem BMBF (Biotechnologie 2020+ Strukturvorhaben: Leibniz Research Cluster, 031 A360B) und der Landesgraduiertenförderung Sachsen-Anhalt für die finanzielle Unterstützung.

\section{Literatur}

[1] Ullrich R, Nuske J, Scheibner K et al. (2004) Novel haloperoxidase from the agaric basidiomycete Agrocybe aegerita oxidizes aryl alcohols and aldehydes.

Appl Environ Microbiol 70:4575-4581 


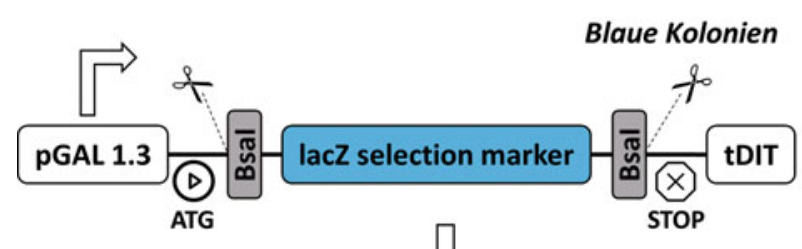

Golden Gate „One pot cloning“ Insertion Transkriptionseinheit
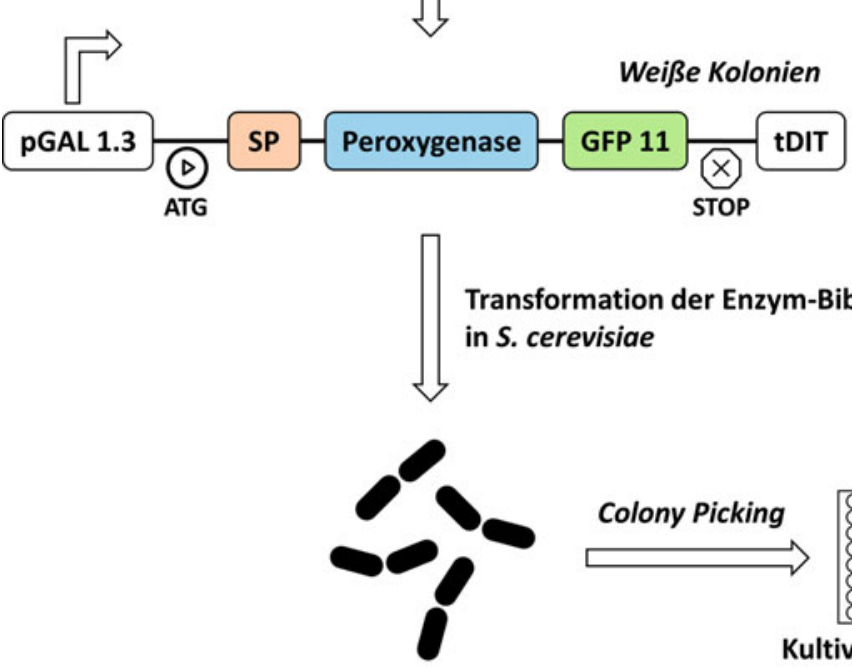

Transformation der Enzym-Bibliothek S. cerevisiae

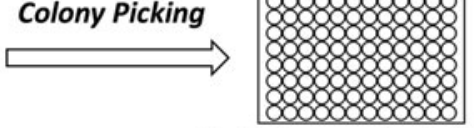

Kultivierung + Induktion

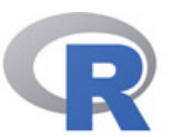

Datenanalyse (Rstudio) Identifizierung von Hits
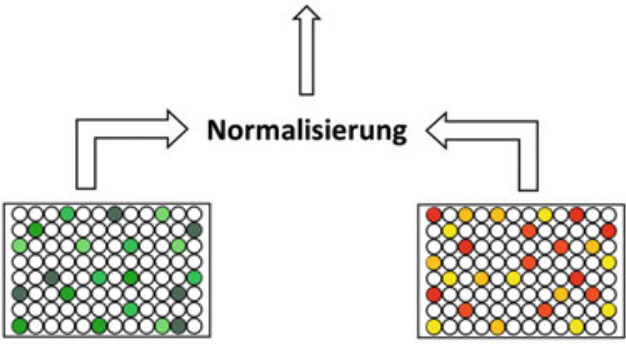

Proteinmenge

Enzymaktivität

Split GFP Assay GC MS Analytik

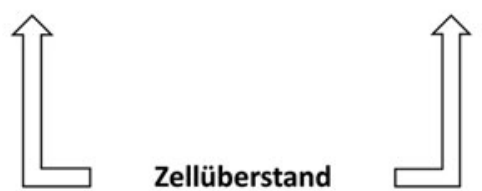

Zellüberstand

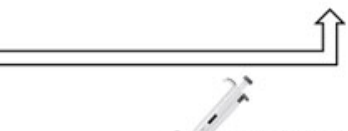

Transferieren

$\Delta$ Abb. 3: Schematische Darstellung des modularen Expressions- und Screeningsystems. Ausgehend von einem entwickelten Golden Gate-kompatiblen Hefe-Expressionsplasmid können Peroxygenase-Transkriptionseinheiten unter Kontrolle eines Galactose-induzierbaren Promotors (pGAL 1.3) innerhalb einer effizienten „one pot"-Golden Gate-Klonierungsreaktion assembliert werden. Die Transkriptionseinheit wird dabei aus drei Teilen gebildet: (1) ein Signalpeptid (SP), (2) das jeweilige Peroxygenase-Gen und (3) der C-terminale GFP-11-Protein-Tag, der eine einfache Proteindetektion im Mikrotiterplattenformat erlaubt. Die der Golden Gate-Reaktion nachfolgende Transformation der Peroxygenase-Bibliothek in Escherichia coli wird als Plasmidbibliothek präpariert, um die erzeugte genetische Diversität zu bewahren. Abschließend wird die Bibliothek in den Expressionsorganismus Saccharomyces cerevisiae transformiert. Die resultierenden Peroxygenase-Varianten werden in 96-Well-Mikrotiterplatten unter Zugabe von Galactose produziert. Nach erfolgter Produktion wird der Zellüberstand auf zwei Mikrotiterplatten aufgeteilt und ein split-GFP-Assay sowie eine Aktivitätsmessung hinsichtlich eines spezifischen Substrats durchgeführt und nachfolgend normalisiert, ein Schritt der die Differenzierung zwischen Aktivität und Proteinkonzentration erlaubt.

[2] Churakova E, Kluge M, Ullrich R et al. (2011) Specific photobiocatalytic oxyfunctionalization reactions.

Angew Chem Int Ed 50:10716-10719

[3] Peter S, Kinne M, Wang X et al. (2011) Selective hydroxy-

lation of alkanes by an extracellular fungal peroxygenase.

FEBS J 278:3667-3675

[4] Ni Y, Fernandez-Fueyo E, Gomez Baraibar A et al. (2016) Peroxygenase-catalyzed oxyfunctionalization reactions promoted by the complete oxidation of methanol.

Angew Chem Int Ed 55:798-801

[5] Faiza M, Huang S, Lan D et al. (2019) New insights on unspecific peroxygenases: superfamily reclassification and evolution. BMC Evol Biol 19:76

[6] Molina-Espeja P, Garcia-Ruiz E, Gonzalez-Perez D et al. (2014) Directed evolution of unspecific peroxygenase from Agrocybe aegerita. Appl Environ Microbiol 80:3496-3507 [7] Püllmann P, Ulpinnis C, Marillonnet S et al. (2019) Golden Mutagenesis: an efficient multi-sitesaturation mutagenesis approach by Golden Gate cloning with automated primer design. bioRxiv:453621

[8] Engler C, Kandzia R, Marillonnet S (2008) A one pot, one step, precision cloning method with high throughput capability. PLoS One 3:e3647
[9] Santos-Aberturas J, Dorr M, Waldo GS et al. (2015) Indepth high-throughput screening of protein engineering libraries by split-GFP direct crude cell extract data normalization. Chem Biol 22:1406-1414

[10] Kiebist J, Schmidtke KU, Zimmermann J et al. (2017) A peroxygenase from Chaetomium globosum catalyzes the selective oxygenation of testosterone. ChemBioChem 18:563-569 [11] Babot ED, Del Rio JC, Canellas M et al. (2015) Steroid hydroxylation by basidiomycete peroxygenases: a combined experimental and computational study. Appl Environ Microbiol 81:4130-4142

Open Access:

Dributed under the terms of the Creative Commons Attribution 4.0 International License (http://creativecommons.org/licenses/by/4.0/), which permits use, duplication, adaption, distribution and reproduction in any medium or format, as long as you give appropriate credit to the original author(s) and the source, provide a link to the Creative Commons license, Opendicate changes were made.

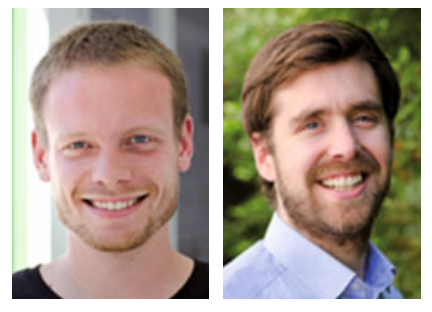

Pascal Püllmann (links) und Martin J. Weissenborn

Korrespondenzadresse:

Jun.-Prof. Dr. Martin Weissenborn Junior Research Group Bioorganic Chemistry Leibniz-Institut für Pflanzenbiochemie (IPB) Institut für Chemie

Martin-Luther-Universität Halle-Wittenberg

Weinberg 3

D-06120 Halle (Saale)

Tel.: 0345-5582-1700

martin.weissenborn@ipb-halle.de

www.ipb-halle.de 